\title{
Comparative analysis of Wnt expression identifies a highly conserved developmental transition in flatworms
}

\author{
Uriel Koziol ${ }^{1,2^{*}}$, Francesca Jarero ${ }^{3}$, Peter D. Olson ${ }^{3}$ and Klaus Brehm ${ }^{2^{*}}$
}

\begin{abstract}
Background: Early developmental patterns of flatworms are extremely diverse and difficult to compare between distant groups. In parasitic flatworms, such as tapeworms, this is confounded by highly derived life cycles involving indirect development, and even the true orientation of the tapeworm antero-posterior (AP) axis has been a matter of controversy. In planarians, and metazoans generally, the AP axis is specified by the canonical Wnt pathway, and we hypothesized that it could also underpin axial formation during larval metamorphosis in tapeworms.
\end{abstract}

Results: By comparative gene expression analysis of Wnt components and conserved AP markers in the tapeworms Echinococcus multilocularis and Hymenolepis microstoma, we found remarkable similarities between the early stages of larval metamorphosis in tapeworms and late embryonic and adult development in planarians. We demonstrate posterior expression of specific Wnt factors during larval metamorphosis and show that scolex formation is preceded by localized expression of Wnt inhibitors. In the highly derived larval form of E. multilocularis, which proliferates asexually within the mammalian host, we found ubiquitous expression of posterior Wnt factors combined with localized expression of Wnt inhibitors that correlates with the asexual budding of scoleces. As in planarians, muscle cells are shown to be a source of secreted Wnt ligands, providing an explanation for the retention of a muscle layer in the immotile E. multilocularis larva.

Conclusions: The strong conservation of gene expression between larval metamorphosis in tapeworms and late embryonic development in planarians suggests, for the first time, a homologous developmental period across this diverse phylum. We postulate these to represent the phylotypic stages of these flatworm groups. Our results support the classical notion that the scolex is the true anterior end of tapeworms. Furthermore, the up-regulation of Wnt inhibitors during the specification of multiple anterior poles suggests a mechanism for the unique asexual reproduction of $E$. multilocularis larvae.

Keywords: Antero-posterior axis, Cestodes, FoxQ2, Metamorphosis, Myocyte, Phylotypic, Planarian, Platyhelminthes, SFRP, Six $3 / 6$, Wnt

\section{Background}

Flatworms (Platyhelminthes) are a highly diverse and ubiquitous phylum of dorso-ventrally flattened animals that include a wide range of free-living and symbiotic forms. The majority of described species are obligate parasites belonging to the Neodermata, a monophyletic group that includes monogeneans, trematodes (flukes), and cestodes

\footnotetext{
* Correspondence: ukoziol@gmail.com; kbrehm@hygiene.uni-wuerzburg.de ${ }^{1}$ Sección Bioquímica, Facultad de Ciencias, Universidad de la República, Montevideo, Uruguay

${ }^{2}$ University of Würzburg, Institute for Hygiene and Microbiology, Würzburg, Germany

Full list of author information is available at the end of the article
}

(tapeworms). The singular origin of neodermatan worms represents one of the most successful evolutionary transitions to parasitism seen in the animal kingdom [1-3].

The Neodermata have complex life cycles with one or more larval stages. Tapeworms (Cestoda) are particularly derived in their morphology and development, as they lack a gut and any trace of endoderm during embryogenesis [4]. The early embryonic development of tapeworms consists of asynchronous and asymmetric divisions, and there is no process resembling gastrulation [5-7]. The final product of embryogenesis is a highly reduced larval form, the oncosphere $[7,8]$. This larva is passively taken up by an intermediate host, and is specialized for 
penetrating through the gut wall by means of six moving hooks and the secretions of penetration glands. The oncosphere metamorphoses into the next life stage, the metacestode, in a parenteral site of the first host. Most of the oncospheral cells are discarded, and the metacestode tissues are generated from a few stem cells that were set aside during embryogenesis $[5,8]$. The metacestode consists of an anterior end, the scolex, which contains attachment organs, and a posterior undifferentiated body. Once the metacestode is ingested by the definitive host, it attaches to the wall of the gut, and develops into the adult form by continuously generating segments from the neck region behind the scolex. Within each segment, male and female reproductive systems develop, resulting in the generation of eggs by sexual reproduction.

Because of the unique morphology and development of tapeworms, it has been impossible to make meaningful comparisons with other flatworms, let alone other animal phyla. Even the true polarity of the tapeworm anteroposterior (AP) axis has been a matter of controversy $[9,10]$. Conventionally, the scolex is taken to be the anterior of the adult, based on its functional orientation and on the centralization of the nervous system within it. However, it has been postulated that this accumulation of nervous elements could simply be due to the requirements for innervation of the muscular attachment organs of the scolex. Furthermore, the relative position of the male and female gonads in each segment (male gonads closer to the scolex) would be opposite that found in freeliving flatworms if the scolex is considered anterior. Perhaps the most controversial point involves the relative polarity of the oncosphere and the adult; during metamorphosis, the scolex is formed at the pole of the oncosphere opposite to that containing the hooks (the "functional anterior end" of the oncosphere, Fig. 1), and therefore it has been postulated that a reversal of polarity occurs during this metamorphosis $[8,9]$.

It has recently been shown (free-living flatworms) that canonical Wnt $/ \beta$-catenin signaling is involved in the specification and maintenance of the AP axis in planarians during regeneration and during normal tissue turnover, similar to what happens during early development in most bilaterian animals [11-15]. Specific Wnt ligands are expressed in gradients from the posterior end, and signaling through this pathway is required to maintain and specify the posterior of adult planarians. Conversely, extracellular inhibitors of Wnts are specifically expressed in the anterior end, and inhibition of canonical Wnt signaling is necessary for anterior specification.

We speculated that, although early development is very divergent between tapeworms and planarians, conservation of a fundamental AP specification program should be shared across the phylum. Thus, the later stages of planarian development should show homologous gene expression patterns to the oncosphere-to-metacestode metamorphosis in tapeworms, as these are the stages where the main body plan is established, whereas earlier developmental stages are specialized for their specific ecological requirements $[8,16]$.

In this work, we elucidate the expression patterns of Wnt ligands and inhibitors as well as other highly conserved animal markers of AP polarity during metacestode development in the tapeworms Echinococcus multilocularis and Hymenolepis microstoma. Our results show a striking conservation of gene expression, leading us to propose that these stages represent the phylotypic period of flatworms, and that the scolex is the true anterior end of tapeworms. Furthermore, our results indicate that the

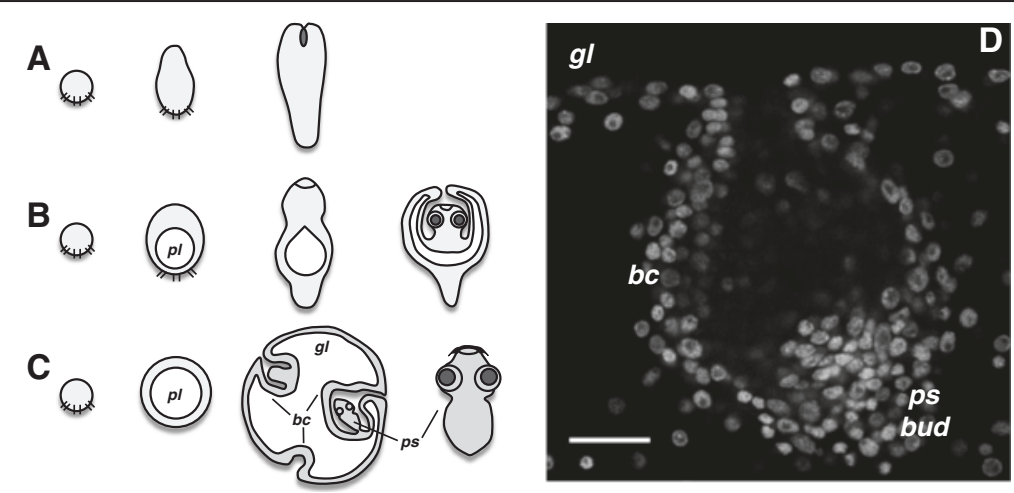

Fig. 1 The oncosphere to metacestode metamorphosis in different tapeworms. A generalized summary of development is shown for different tapeworm groups. a Development in basal eucestodes, without the formation of an inner cavity. b Development in most cyclophyllidean tapeworms, with the formation of a cavity (primary lacuna, $p$ ) during early development. The scolex invaginates or retracts into this cavity during later development. c Development in Echinococcus multilocularis. The tissues surrounding the primary lacuna expand into the thin germinal layer (g), from which many brood capsules (bc) invaginate. The protoscoleces ( $p s)$ develop within the brood capsules, and are released when the metacestode is ingested by the definitive host. $\mathbf{d}$ Nuclear staining with 4',6-diamidino-2-phenylindole (DAPI) of an E. multilocularis metacestode vesicle from in vitro culture, showing the continuity of the germinal layer ( $($ )), brood capsule (bc) and protoscolex bud (ps bud). Bar: $20 \mu \mathrm{m}$ 
unique development of the E. multilocularis metacestode is the result of a lack of AP polarity during early development, resulting in a completely posteriorized metacestode from which multiple foci of anterior development subsequently arise.

\section{Results}

A re-interpretation of $E$. multilocularis larval morphology and development

The life cycle outlined in the introduction is a generalization of the diversity found in the 'true' tapeworms, the Eucestoda (Fig. 1a). However, variations of this plan occur in particular groups. Many species of the order Cyclophyllidea form an internal cavity during early metacestode development, called the primary lacuna (Fig. 1b) $[8,17,18]$. The tissues surrounding this cavity form a cyst or bladder. The scolex retracts or invaginates into this cavity, and is protected by the surrounding tissue (Fig. 1b).

Among cyclophyllideans, metacestodes of Echinococcus spp. are the causative agents of dangerous zoonoses worldwide [19], and display unique development in their intermediate hosts (typically in the liver) (Fig. 1c) [17, 20]. Initially, only bladder tissue is generated from the oncosphere, forming a large vesicle that is filled with fluid, and contains only a thin layer of tissue in the periphery (the germinal layer) (Fig. 1c). From the germinal layer, secondary vesicles, called brood capsules, are formed towards the inner cavity. Within the brood capsules, nascent scoleces (protoscoleces) are formed by budding, resulting in massive asexual propagation (Fig. 1c). The protoscoleces are attached to the brood capsule by a thin stalk, and are released when the metacestode is ingested by the definitive host.

The evolutionary origin of the unique E. multilocularis metacestode remains unsolved. This is partly because the development of E. multilocularis has been historically regarded to be fundamentally different from that of other tapeworms, as the protoscoleces were considered to form within the central cavity, towards its interior (endogenous development), as opposed to forming on the external surface of the metacestode as in most other tapeworms (exogenous development) [8, 21-23]. However, we have recently shown, using confocal microscopy, that brood capsules and protoscoleces of E. multilocularis are actually formed from an invagination of the germinal layer of metacestode vesicles, and therefore just as in other tapeworms, the scolex is formed from the metacestode body wall towards the exterior [24] (Fig. 1c,d; see also $[25,26])$. If one assumes that the scolex is the anterior end of tapeworms, then the E. multilocularis metacestode can be interpreted as showing differentiation along an AP axis, with many anterior ends (protoscoleces) followed along the AP axis by the brood capsules, and finally converging to one common posterior represented by the germinal layer of the vesicles. Therefore, we hypothesized that, during early development, the $E$. multilocularis metacestode is composed exclusively of posterior tissues and anterior development is suppressed. The remaining stages of metacestode differentiation are delayed, and only later do multiple foci of anterior development arise from the germinal layer. This is in contrast to most tapeworms, in which a single scolex forms very early during development, always in the region opposite to the hooks of the oncosphere [8].

\section{Expression of Wnt and SFRP genes during early metacestode development of $E$. multilocularis}

We analyzed the expression of homologs of Wnt ligands and of inhibitors of Wnt signaling involved in planarian AP specification using whole-mount in situ hybridization (WMISH) [11, 12, 14, 15, 27] (Figs. 2 and 3). Although tapeworms have lost many developmental genes conserved in most animals, they possess clear homologs of all families of Wnt ligands present in planarians [28, 29]. They also have one clear member of the SFRP family of Wnt inhibitors containing a cysteine-rich domain and a netrin domain [28], and another member with a divergent netrin domain dubbed SFRP-like. In planarians, three different SFRP genes are expressed in overlapping anterior domains of which sfrp-1 is considered a bona fide marker of anterior specification during regeneration $[11,14,27,30]$.

Strikingly, the E. multilocularis em-sfrp and em-sfl genes are not expressed in the germinal layer of the metacestode (they cannot even be detected by RT-PCR), and expression first appears when brood capsule buds develop as small accumulations of cells protruding from the germinal layer (Fig. 2). Throughout the development of the brood capsules and the protoscoleces, em-sfrp is expressed in the anterior-most region, eventually becoming restricted to a few cells at the tip of the developing protoscolex (Fig. 2). em-sfl also shows anterior expression but is less restricted, with strong expression in the apical end of the protoscolex but also in the protoscolex body and in the brood capsule (Fig. 2). These genes are the earliest known markers of brood capsule development. Conversely, when we analyzed the expression of homologs of posterior Wnt genes from planarians (em-wnt1, em-wnt11A and em$w n t 11 B)$, they were all expressed in dispersed cells in the germinal layer of the metacestode vesicles, and during brood capsule and protoscolex development they were always expressed in posterior domains: em-wnt 1 and em$w n t 11 B$ were always restricted to the germinal layer and to the base of the brood capsule, whereas em-wnt11A was expressed throughout the germinal layer and brood capsule, and also at the posterior end of the developing protoscolex (Fig. 2). These results are not only compatible with our hypothesis, but also suggest that the formation of 


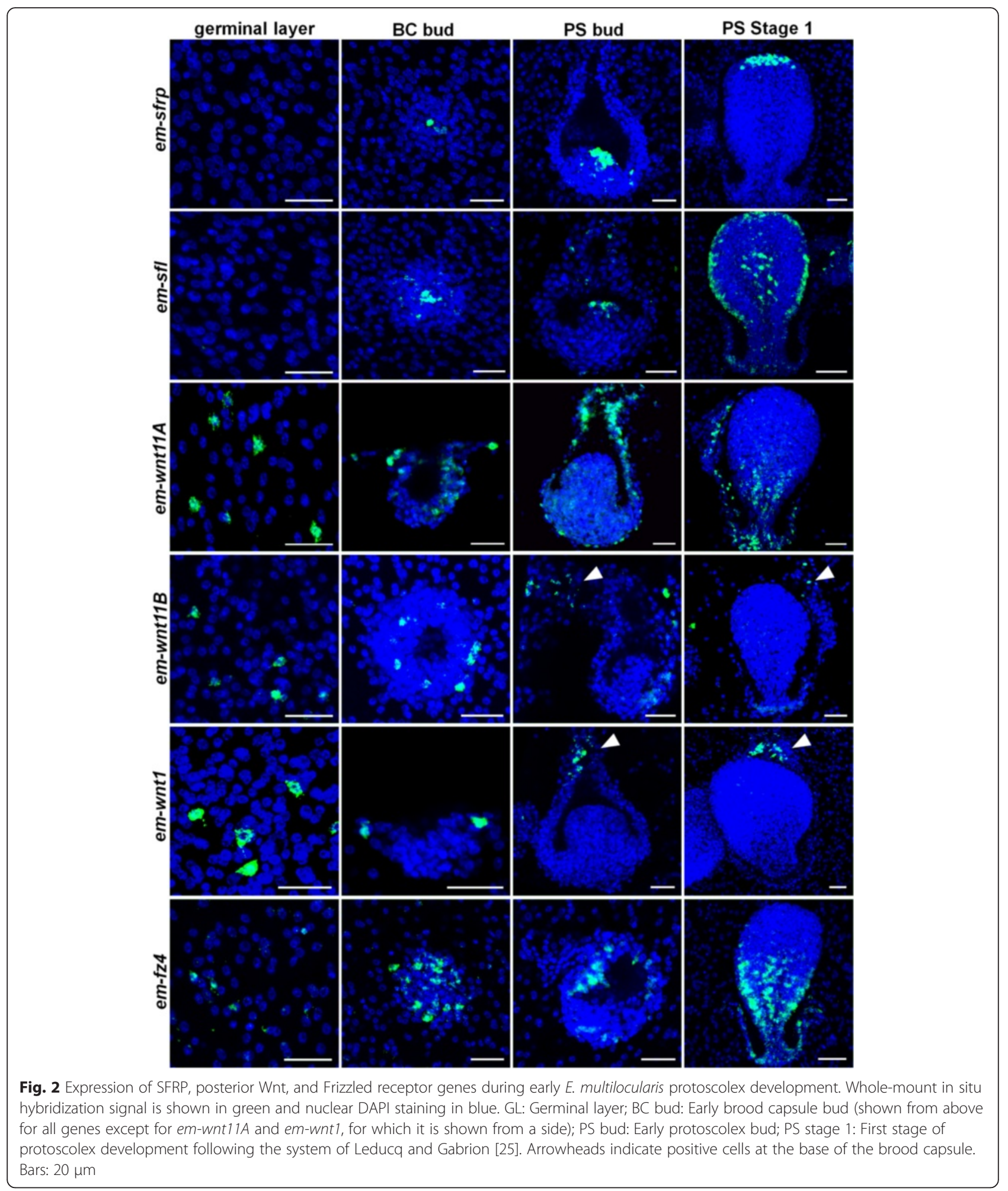

brood capsules is induced by the specific inhibition of posterior Wnt ligands that are widespread in the germinal layer. They also correspond remarkably to expression patterns in planarians, in which SFRP and Wnt genes are expressed in anterior and posterior overlapping domains, respectively, with wnt1 being the most posterior [12, 27].

Encouraged by these results, we analyzed the expression patterns of the remaining Wnt genes in E. multilocularis 


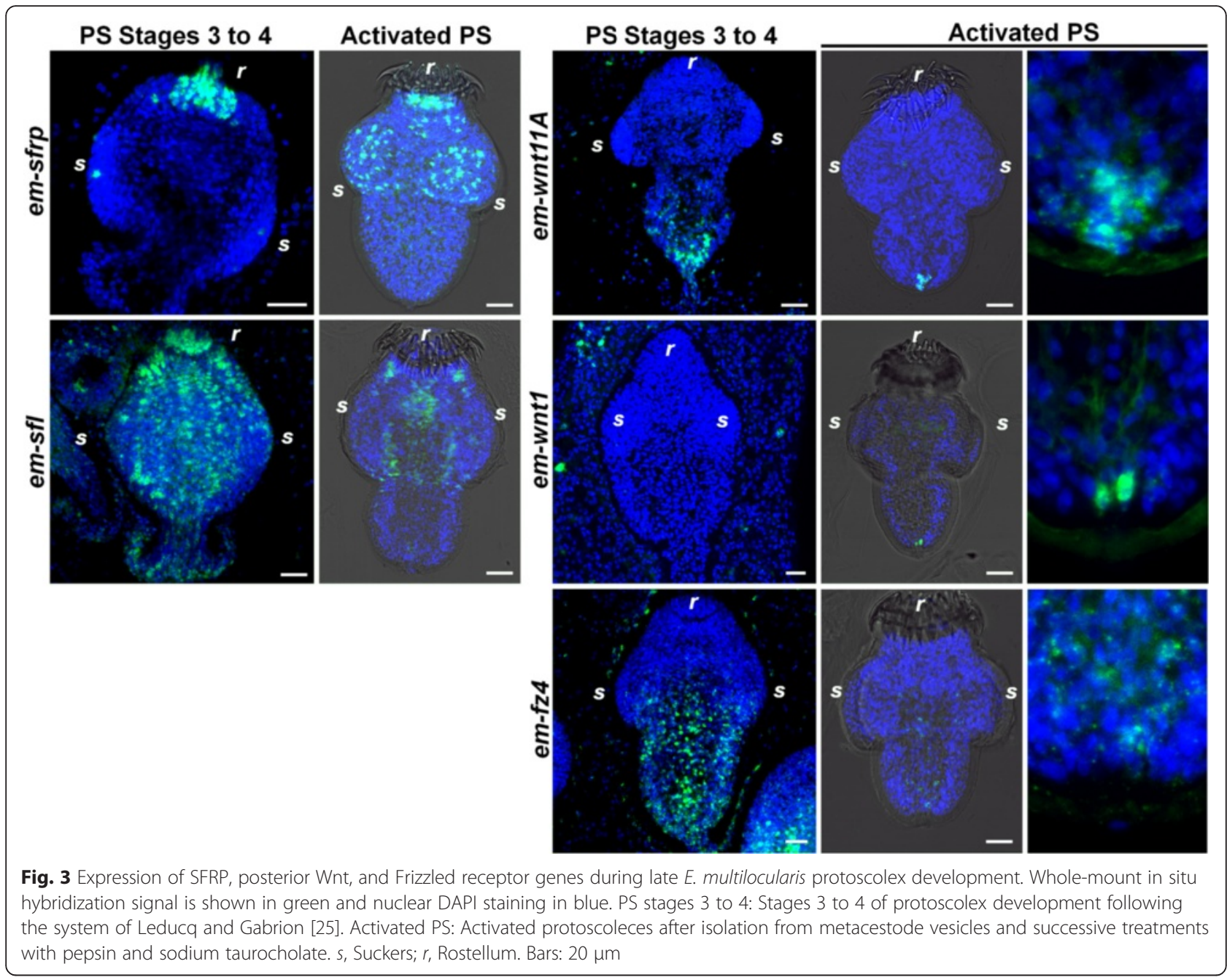

metacestodes (Fig. 4). The wnt2 gene of planarians is expressed in two antero-lateral domains that surround the apical $s f r p 1^{+}$cells $[14,27]$. We see a remarkably similar expression pattern of em-wnt2, since it is not expressed at all in the germinal layer or brood capsules, and expression appears in two antero-lateral domains of the developing protoscolex which surround the em-sfrp ${ }^{+}$ expression domain. On the other hand, wnt5 of planarians is expressed in lateral domains in planarians and is involved in the specification of the mediolateral axis through non-canonical Wnt signaling [27, 31]. Once again, we observe an equivalent pattern in the developing protoscolex, in which em-wnt5 is expressed as two lateral stripes (Fig. 4). Unfortunately, we have not been able to obtain reproducible WMISH results for the remaining wnt gene, em-wnt4.

Finally, we analyzed the expression of em-fz4, an ortholog of a planarian Frizzled receptor expressed in the posterior-most region of the body and used as a specific posterior marker (named $f z 4, f z T$ or $f z$-d by different authors in different planarian species [11, 12, 32-34])
(Additional file 1). Frizzleds are a family of receptors for Wnt ligands that participate in both canonical and noncanonical signaling $[35,36]$. The $e m-f z 4$ gene is expressed in dispersed cells in the germinal layer, becomes strongly up-regulated during brood capsule development, and is always restricted to the posterior-most region of the protoscolex throughout development (Fig. 2).

\section{Expression patterns during later E. multilocularis protoscolex development}

We also analyzed the expression of these genes during later protoscolex development, including completely developed protoscoleces, which had been activated by mimicking the infection of the definitive host. For some genes, the expression patterns did not vary significantly (for example, em-wnt2 and em-wnt5; Fig. 3). For em-wnt11a, expression was restricted to the posterior region of the protoscolex throughout, becoming progressively more restricted and only being expressed in a few posterior-most cells in the developed protoscolex (Fig. 3). The em-wnt1 and em-wnt11b genes are always restricted 


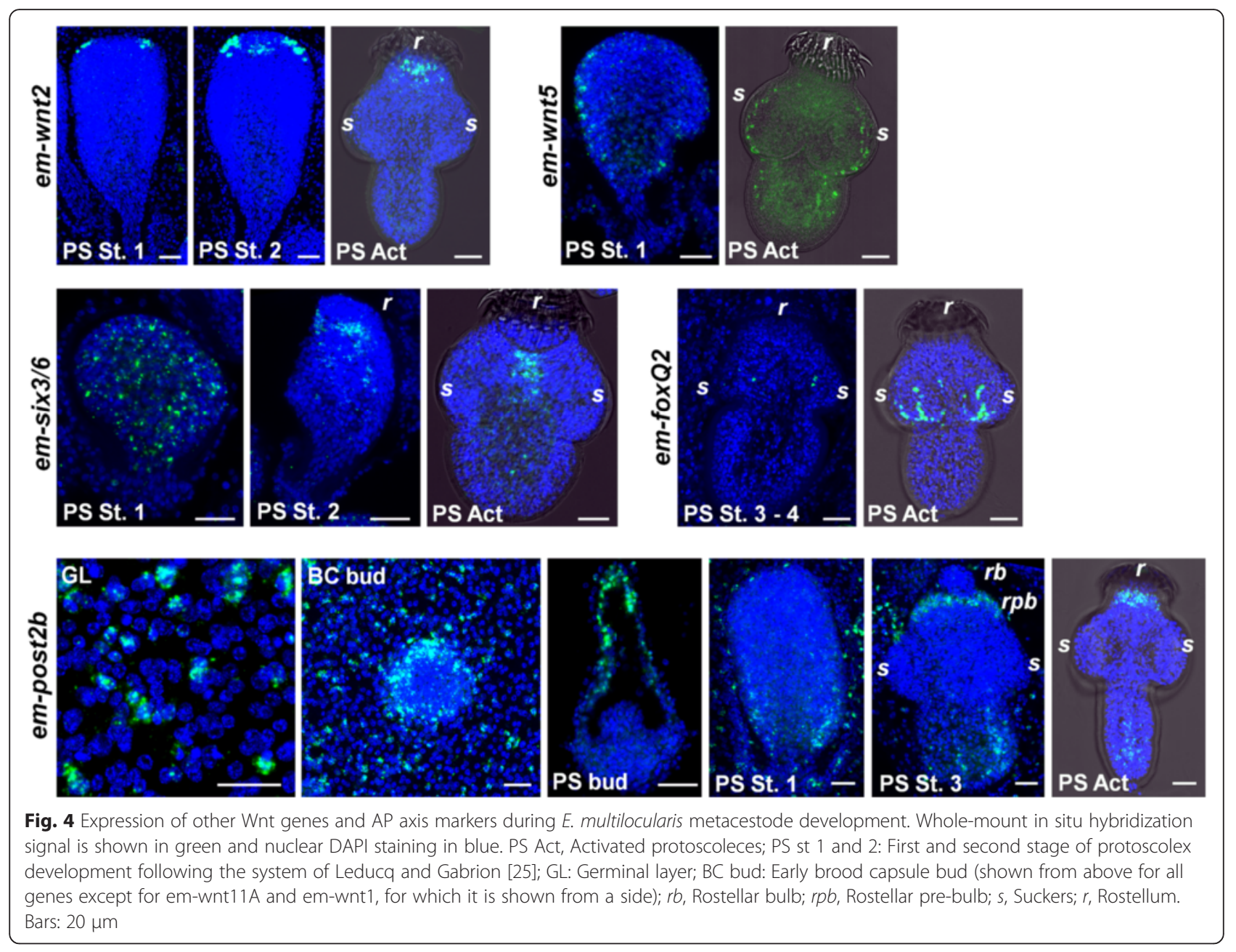

to the base of the brood capsule and are not expressed in the protoscolex itself (Fig. 3 and data not shown). Interestingly, after protoscolex release and activation, em-wnt1 also becomes expressed in the posterior-most cells, similar to em-wnt11a (Fig. 3). Finally, em-fz4 is always restricted to the posterior of the protoscolex and becomes barely detectable after development is complete (Fig. 3).

For other genes, the original expression patterns remained but additional sites of expression could be detected as development progressed. In the case of em-sfrp, the apical domain of expression persists throughout development, including in the apical rostellum (a muscular attachment organ containing hooks). However, during later development, new foci of expression appear in the scolex at each of the four developing sucker primordia, and expression remains in the suckers of the completely developed protoscolex (Fig. 3). In the case of em-sfl, it was later expressed in many tissues during protoscolex development, but was restricted to the tissues surrounding the rostellum in the completely developed protoscolex (Fig. 3).
In summary, expression patterns observed during early developmental stages are shown to be largely maintained in later stages, but new expression domains appear that are not directly comparable to those of planarians. This is compatible with the hypothesis that early metacestode metamorphosis is the most highly conserved stage of development, and developmental gene expression diverges in later stages as tapeworm-specific characters, such as the attachment organs, are formed.

\section{Expression of conserved AP markers in E. multilocularis}

In order to further test our hypothesis, we analyzed the expression patterns of AP markers that are well conserved in bilaterian animals but are not directly related to Wnt signaling. Our choices of markers were limited as many such genes have been lost in tapeworms or are too divergent to be identified unambiguously [29]. As a classical anterior maker, we have chosen the homeobox gene Six $3 / 6$ which is expressed in the anterior of bilaterian embryos (especially in the anterior-most region of the nervous system) [37]. In planarians, six $3 / 6$ is expressed in 
the outer and anterior-most region of the brain [38, 39]. A clear ortholog of $s i x 3 / 6$ is present in tapeworms (Additional file 2). In E. multilocularis, em-six3/6 is not expressed in the germinal layer. Low levels of expression first appear throughout the early protoscolex buds, and the expression domain of em-six $3 / 6$ is progressively restricted during protoscolex development to the region behind the developing rostellum (Fig. 4). This area represents the rostellar nerve ring, the most anterior region of the central nervous system [24]. We also analyzed the expression of an ortho$\log$ of foxQ2 (Additional file 3), which is expressed in the anterior-most region of many animals [40-42]. However, the planarian ortholog is expressed in the brain but not in the anterior-most region [39], and similarly, in E. multilocularis em-foxQ2 appears to be expressed in the nervous system of the scolex, but expression only occurs during late development in the region where the nervous system associates with the suckers (postero-lateral ganglia and sucker nerve rings [24]; Fig. 4).

Hox genes have conserved roles in the specification of body regions along the AP axis in bilaterian animals [43]. As a classical posterior marker, we chose the posterior Hox gene post2, a homolog of which is also expressed in the posterior body of planarians $[15,44]$. The E. multilocularis ortholog em-post $2 b$ [45] is strongly expressed in the germinal layer and brood capsule, and is restricted to the posterior most regions of the protoscolex during early development, thus supporting our hypothesis (Fig. 4). At later stages of protoscolex development, a second expression domain also appears in the rostellar pre-bulb region, which forms the rostellar hooks. This expression domain becomes dominant in the completely developed protoscolex (Fig. 4).

Therefore, once again, we observe comparable gene expression patterns in early metacestode development of E. multilocularis and in adults of planarians. During later metacestode development, divergent expression patterns appear, which are related to tapeworm-specific morphological innovations with no clear counterpart in planarians.

\section{Expression of Wnt and SFRP genes during Hymenolepis microstoma metamorphosis}

Our results regarding gene expression in E. multilocularis strongly support our hypothesis that conserved gene expression patterns can be found during the larval metamorphosis of tapeworms. However, the development of $E$. multilocularis metacestodes is highly derived. Therefore, we wished to determine if similar gene expression patterns are also present in tapeworms with a more primitive form of development, and for this we chose the well-established model $H$. microstoma [46]. In $H$. microstoma, as in nearly all tapeworms, the oncosphere gives rise to a single juvenile worm with a scolex that develops at the pole opposite of the larval hooks (Fig. 1b). The polarity of the oncosphere is therefore reflected in the AP axis of the metacestode. However, like E. multilocularis and most other cyclophyllidean tapeworms, a primary lacuna (i.e. cavity) forms [47], later collapsing and encysting the nascent tapeworm.

Clear orthologs of all the described Wnt and SFRP genes of E. multilocularis are present in H. microstoma, and were identified with the same name, together with an $h m$ prefix. As early as 48 hours after ingestion by the intermediate host, expression of the Wnt inhibitor hm-sfrp appears at the pole that will give rise to the scolex and is maintained throughout metamorphosis (Fig. 5). The hm$s f l$ gene is initially expressed apically, and later extends along the anterior region in two lateral stripes that begin sub-apically and end short of the opposite pole. In both cases, expression domains mirror those of E. multilocularis during protoscolex formation within the brood capsules (Fig. 2). Expression of 'posterior' Wnts hm-wnt1, $h m$-wnt11a and $h m$-wnt11b, and the frizzled receptor $h m$ frzd4 is largely ubiquitous at the onset of metamorphosis, but becomes increasingly restricted to the posterior as the cells of the apical pole proliferate and condense to form the anterior regions. In addition, Wnts hm-wnt11a and $h m-w n t 11 b$ show strong expression in two cells that mark the posterior pole, and hm-wnt11a also shows lateral expression in the central portion of the larvae (Fig. 5 and Additional file 4). Discrete expression of hm-wnt11a and $h m-w n t 11 b$ at the posterior pole in Hymenolepis mirrors that seen in E. multilocularis during protoscolex development (Fig. 3). Thus, like in E. multilocularis, the metamorphosis of the $H$. microstoma larva begins with ubiquitous expression of Wnt ligands, from which a pole of Wnt inhibition leads to anterior development. The expression of genes coding for posterior Wnt ligands further supports the homology of the E. multilocularis germinal layer and the tissues that encyst the Hymenolepis juvenile. Other Wnt genes also replicate the expression domains observed in E. multilocularis: hm-wnt2 is initially expressed in two anterolateral foci, which later expand slightly to surround the developing rostellum, whereas $h m-w n t 5$ is expressed in the lateral margins throughout larval development (Fig. 6 and Additional file 5). Taken together, the expression domains of these genes corroborate the expression of Wnt components seen in both tapeworm and planarian flatworms. They also help to confirm homologies between the convoluted morphology of E. multilocularis larvae and more typical tapeworm larval forms.

\section{Muscle cells are a source of Wnts in the E. multilocularis germinal layer}

In planarians, Wnts, SFRPs, and other genes related to the specification of the body axes (the so-called 'position control genes', PCGs) are expressed by muscle cells in the body wall, providing positional information during 


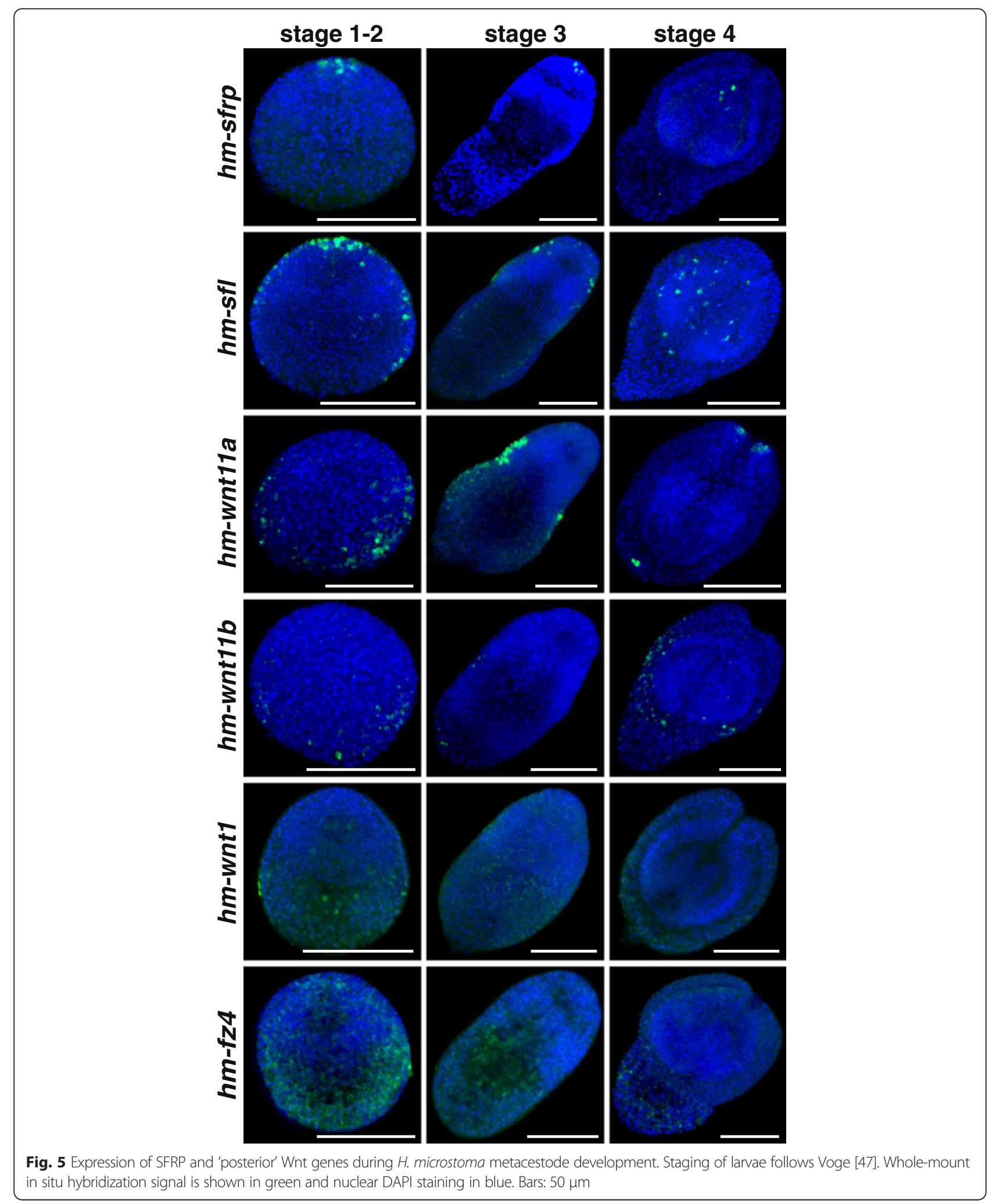

normal tissue turnover and regeneration [48]. The products of PCGs influence the behavior of neoblasts, which are pluripotent stem cells that do not express PCGs.
In tapeworms, similar stem cells (often called germinative cells) exist [49]. We have recently characterized the stem cells of E. multilocularis, and demonstrated that 


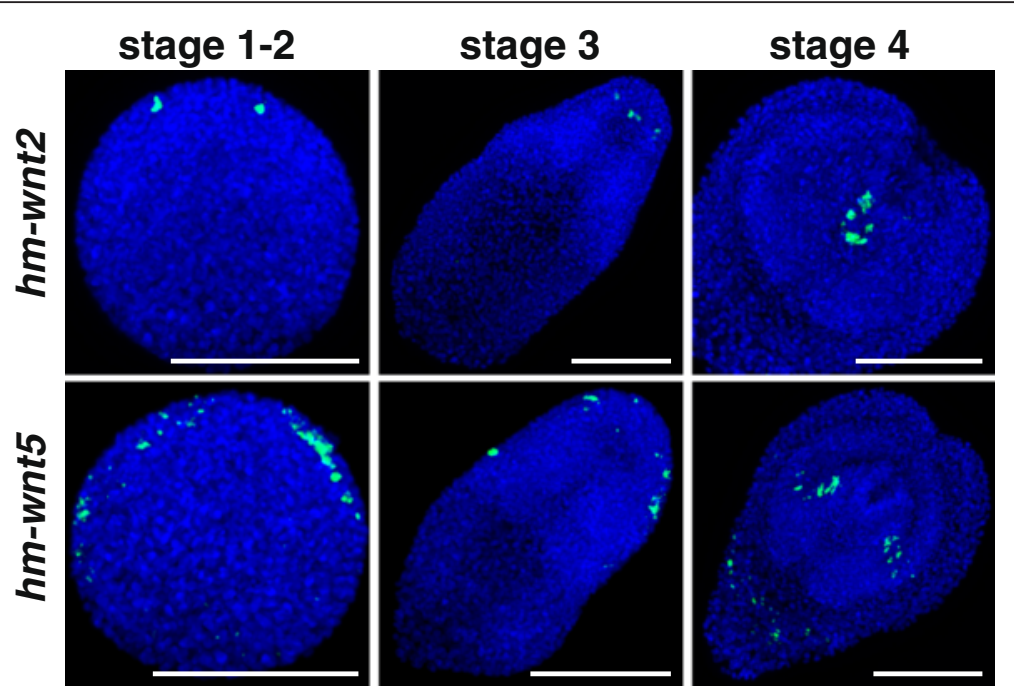

Fig. 6 Expression of other Wnt genes during H. microstoma metacestode development. Staging of larvae follows Voge [47]. Whole-mount in situ hybridization signal is shown in green and nuclear DAPI staining in blue. Bars: $50 \mu \mathrm{m}$

these cells are the only proliferative cells, as is the case in all flatworms studied to date [50]. Therefore, they can be specifically labeled by incubation with thymidine analogs such as 5-ethynyl-2'-deoxyuridine (EdU), which are incorporated into DNA during replication [50]. In order to determine if Wnt and SFRP genes are expressed by stem cells, we performed double labeling of WMISH for each gene together with the detection of EdU incorporation. We observed little to no incorporation of EdU in cells positive for em-wnt1 (0.0\%; $\mathrm{n}=148$ cells $)$, em-wnt11a ( $2.2 \% ; \mathrm{n}=716$ cells), and em-wnt11b (0.8 \%; $\mathrm{n}=241$ cells) in the germinal layer (Fig. 7). This was also the case in developing brood capsules and protoscoleces for em-wnt1 ( $0.3 \% ; \mathrm{n}=377$ cells), em-wnt11a ( $1.6 \%, \mathrm{n}=1,150$ cells $)$, em-wnt11b (0.7 \%; $\mathrm{n}=241$ cells $)$ and em-sfl ( $0.2 \% ; \mathrm{n}=426$ cells) (Fig. 7). Furthermore, em-sfrp is only expressed in the post-mitotic apical region [50]. Therefore, as in planarians, there is no significant expression of PCGs in tapeworm stem cells.

In the germinal layer of $E$. multilocularis vesicles, there are muscle cells that form a layer of disorganized muscle fibers. In tapeworms, the contractile muscle fibers (myofibers) are only connected by thin cytoplasmic strands to the main cell body (myocyton) containing the nucleus and most organelles [51]. We performed immunohistofluorescence of metacestode vesicles with an antitropomyosin (TPM) antibody that specifically labels the muscle fibers of tapeworms, including those of E. multilocularis [50, 52, 53]. Besides the strong signal in large muscle fibers, in many cases we could also observe thin $\mathrm{TPM}^{+}$filaments in the cytoplasm of myocytons that converged into myofibers (Fig. 8). By double labeling, we observed that many cells expressing em-wnt1 and em$w n t 11 a$ in the germinal layer are muscle cells, since they also have $\mathrm{TPM}^{+}$filaments in the surrounding cytoplasm, and the $\mathrm{WMISH}^{+}$cytoplasm was clearly connected to long $\mathrm{TPM}^{+}$myofibers (Fig. 8). Because not all muscle cells showed $\mathrm{TPM}^{+}$fibers in the myocyton, we were unable to determine the percentage of muscle cells expressing each gene. Therefore, posterior Wnts are not only expressed in similar domains in E. multilocularis metacestodes and planarians adults, but also by the same cell type.

\section{Discussion}

The polarity of the tapeworm AP axis is conserved with other bilaterians

Our results clearly support the classical assumption that the scolex represents the true anterior end of tapeworms, and suggest that the condensation of the nervous and osmoregulatory systems present in the scolex are examples of cephalization. One of the main historical controversies regarding the AP axis of cestodes has been the interpretation of the polarity of the oncosphere, and how it relates to the polarity of the metacestode and adult [8]. The pole containing the hooks and the pores of the penetration glands has been considered functionally anterior, but the scolex develops from the opposite pole. However, oncospheres lack a brain (even the presence of nerve cells is controversial), do not move directionally and are taken up passively by the host $[8,54]$. The most basal cestode lineages (Amphilinidea and Gyrocotylidea) have a more complex ciliated larva with ten hooks called the lycophora. This larva has an anterior brain and penetrates actively through the body wall of the intermediate host [55]. Importantly, during swimming the end of the lycophora bearing the hooks is directed posteriorly [56]. In the amphilinidean Austramphilina elongata, once the lycophora larva makes contact with the host with its anterior 


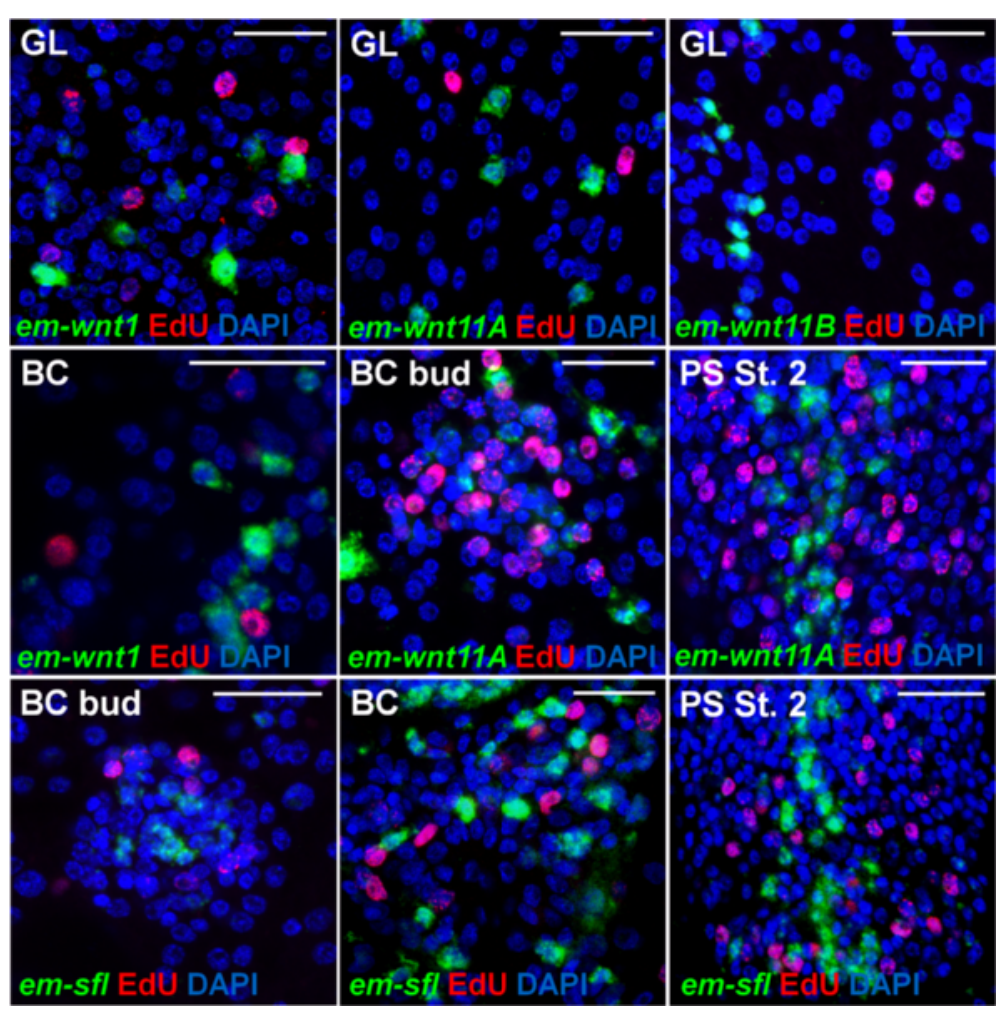

Fig. 7 Lack of EdU incorporation in cells expressing Wnt and SFRP genes in E. multilocularis metacestodes. Green: Whole-mount in situ hybridization (WMISH); red: EdU detection; blue: nuclear DAPI staining. EdU labeling was done by culturing metacestodes in vitro for 5 hours with $50 \mu \mathrm{M}$ EdU. Notice the lack of co-localization between WMISH and EdU for each gene at each stage. GL: Germinal layer; BC bud: Early brood capsule bud (shown from above for all genes except for em-wnt11A and em-wnt1, for which it is shown from a side); PS st 2: Second stage of protoscolex development following the system of Leducq and Gabrion [25]. Bars: $20 \mu \mathrm{m}$

end, it bends into a U-shape that brings the posterior hooks and the anterior end together as the hooks participate in the penetration of the host [55]. During development of the juvenile, the end opposite of the hooks develops into a sucker for attachment [55]. Therefore, in basal cestodes, the functional end of the larva is the same as that of the adult, and the hooks are simply posterior organs for attachment and penetration. Wnt expression corroborates this notion and thus supports an evolutionary transition in which the oncosphere was derived by extensive reduction of a lycophora larva, and the posteriorly-oriented hooks maintained their role for penetration (now through the gut of the host) as eucestodes transitioned to a passive mode of infection of the intermediate host.

Another classical argument opposing the view that the scolex is the anterior pole of tapeworms is the fact that, if the scolex is considered anterior, then the relative position of the male and female gonads (testes anterior to the ovary) would be opposite that found in free-living flatworms. It is therefore possible that a change in the relative position of the gonads in the adult (that is, after the developmental stages covered in this work) occurred during tapeworm evolution. Finally, when the scolex is considered anterior, tapeworms show a form of growth that is contrary to that of all other segmented animals, as new segments are formed in the anterior germinative region in the neck (as opposed to a posterior growth zone as seen, for example, in annelids) [10]. However, tapeworm segmentation evolved de novo in the eucestode lineage and is not homologous to that seen in other animals [3, 57], making developmental comparisons impossible.

\section{A phylotypic stage for flatworms}

The Neodermata and several derived groups of freeliving flatworms have extremely divergent modes of early embryonic development as a consequence of their unique eggs, which contain not only the oocyte, but also specialized cells called vitellocytes that produce yolk and shell proteins $[2,58]$. Different flatworm groups have developed independent solutions to the problem of incorporating the yolk mass into the embryo, resulting in unique early developmental patterns. This is in stark contrast to the 


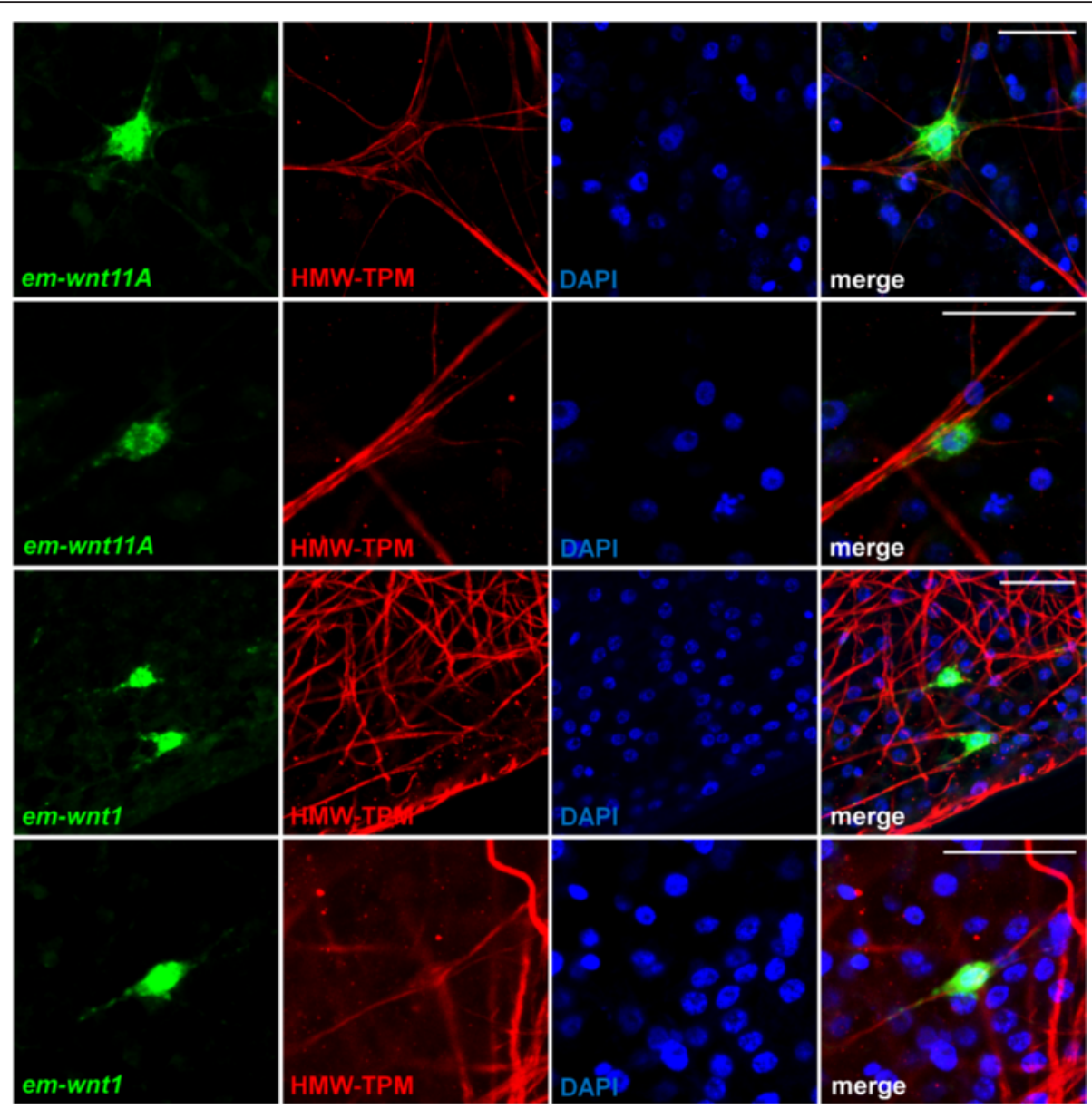

Fig. 8 Expression of Wnt genes in muscle cells in E. multilocularis metacestode vesicles. Green: Whole-mount in situ hybridization; red: immunodetection of high molecular weight tropomyosins; blue: nuclear DAPI staining. For each gene, two examples of positive muscle cells with different morphologies are shown. Bars: $20 \mu \mathrm{m}$

spiral cleavage present in most basal flatworm lineages and also in many other members of the superphylum Lophotrochozoa [58]. Therefore, it is very difficult to search for homologies in the development of different flatworm groups based solely on morphological grounds.

Despite this morphological diversity, we observe that the expression patterns of genes related to AP polarity and other PCGs are strikingly similar in tapeworms during larval metamorphosis and in planarians at late stages of embryonic development (Fig. 9). In contrast, early embryonic stages are highly divergent, and represent specific adaptations to their unique lifestyles. In planarians, early blastomeres disperse into the mass of vitellocytes (in a process aptly named 'blastomere anarchy') and eventually surround it. Later, a transient embryonic pharynx is formed [16]. All of these modifications are a consequence of the need to incorporate the external yolk, arising as an evolutionary adaptation to competition between several embryos within a single egg for limited yolk resources [16, 58]. During early development of planarians, many PCGs are already expressed, but in patterns that do not resemble those in later stages or in other animals, and the relationships between the axes of the early embryo and the adult are not clear [59]. At later stages of development, the definitive regions and tissues of the body of the embryo arise from ventral condensations of blastomeres, and it is at this stage that the PCGs acquire canonical expression patterns [59]. A similar developmental stage has been described in other flatworm groups, and it has been proposed to be the phylotypic stage for free-living flatworms [58]. The phylotypic stage is usually defined as a stage during mid-embryogenesis where the similarity in body plan and corresponding expression of developmental genes is maximal across the species of a phylum, and the definitive body plan of the animal is established [60,61]. The phylotypic stage is expected to be highly conserved due to developmental constraints of numerous global interactions (unlike early 

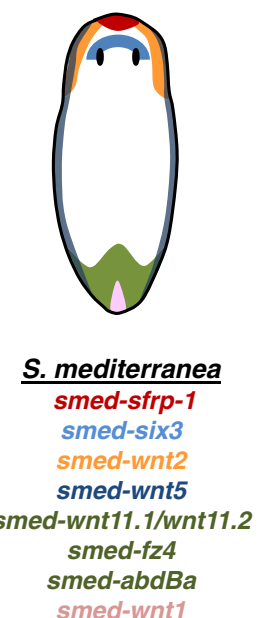

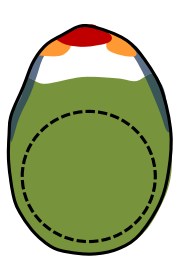

H. microstoma hm-sfrp

hm-wnt2

hm-wnt5

hm-wnt11A

hm-fz4

hm-wnt1

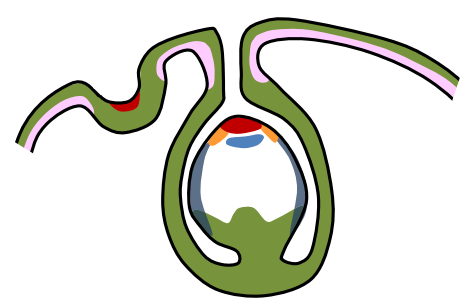

E. multilocularis

em-sfrp

em-six3/6

em-wnt2

em-wnt5

em-wnt11A

em-fz4

em-post2A

em-wnt1

Fig. 9 Comparison of gene expression patterns in adults of the planarian Schmidtea mediterranea, with H. microstoma and E. multilocularis metacestodes. Approximate expression domains are shown with different colors, and in some cases several genes with slightly different expression domains were grouped together. References for the S. mediterranea expression patterns are given in the main text

development, which is highly variable in many taxa) and significant similarities can also be drawn at this stage with gene expression patterns in other animal phyla [60,61].

We propose that the phylotypic stage suggested for free-living flatworms also applies to tapeworms. In the case of tapeworms, early development is also divergent, and the formation of the oncosphere is an evolutionary adaptation for the infection of the first host of the life cycle. The metacestode stage can be considered to have the basic body plan of tapeworms, as in basal cestode groups the only further development that occurs is sexual maturation, and segmentation in eucestodes is an evolutionarily derived novelty imposed over this basic body plan $[3,55,57]$. Therefore, during early metamorphosis of the oncosphere, the body plan is established and it is precisely at this stage that strong conservation is seen in the expression patterns of metazoan developmental genes. Later, development again diverges between groups, as specific organs of attachment are formed, representing the onset of the next ontogenetic phase of the life cycle. The expression of some PCGs diverges at this point, suggesting that they have become exapted for novel roles, in agreement with the idea that only the early metamorphosis represents the phylotypic stage. It would be interesting to determine the expression of PCGs in early tapeworm embryogenesis, but this is technically challenging as the embryo develops within the egg, and in cyclophyllideans also in utero.

In planarians, the anterior pole of the head has been proposed to organize anterior development during adult regeneration and expresses sfrp-1 together with other inhibitors of Wnt signaling [62]. An apical pole of sfrp-1 expression is also the earliest expression pattern of a PCG in the embryo that resembles that found in the adult [59]. Similarly, an apical pole of sfrp expression is observed very early during tapeworm anterior development. Interestingly, this region later becomes the rostellum, and continues to express sfrp. The rostellum is an evolutionary modification of the apical organ, an attachment organ that is formed in all tapeworms during metamorphosis and which may persist in the adult or be only transitory $[4,18]$. Because of the universal conservation of this apical organ in tapeworms, it is possible that it also has a conserved role as an anterior organizer and, in fact, such a role has been previously proposed for the rostellum [63].

\section{Evolutionary origins of the unique E. multilocularis development}

The massive metacestodes of Echinococcus spp. result from extensive growth of the germinal layer. In the related metacestodes of Taenia spp. (that together with Echinococcus spp. comprise the family Taeniidae), the bladder also reaches large proportions, and one or many scoleces develop from the bladder tissue. The morphology of the metacestodes from Taenia spp. can be remarkably varied, including large bladders with a single scolex (cysticercus), with many invaginated scoleces (coenurus), or with many externally protruding scoleces ('polycephalic larvae') $[17,64,65]$, but in all cases the scolex develops towards the exterior of the bladder (exogenous development). Asexual formation of many scoleces is found in many basal taeniid lineages, suggesting that it was present in the last common ancestor of the family, but homology of asexual reproduction in taeniids is controversial [65-69]. Because the development of $E$. multilocularis is actually also exogenous, we propose that there is no fundamental difference in the development of 
taeniid metacestodes, supporting the hypothesis that asexual scolex formation is ancestral in taeniids (and was lost secondarily in several lineages). One unique characteristic of $E$. multilocularis metacestodes is the secretion of a thick external acellular covering, the laminated layer [70]. The brood capsules form as invaginations of the germinal layer that are not accompanied by the laminated layer. Therefore, brood capsules can be regarded as specializations of the germinal layer in which protoscoleces can develop without a laminated layer cover.

The E. multilocularis metacestode initially forms as a hollow cyst that lacks morphological polarity, and shows widespread expression of posterior Wnt genes. This suggests that, in E. multilocularis, anterior development is suppressed during the early stages of the oncosphereto-metacestode transition, and that Wnt signaling is important for maintaining the posterior identity of the tissues. The formation of protoscoleces only occurs after months of infection [21, 71], and is accompanied by the expression of Wnt inhibitors, indicating that suppression of Wnt signaling is important for the formation of anterior structures. In contrast, in other tapeworms, the scolex primordium develops very early (for example, at 48 hours post-infection in $H$. microstoma) as a condensation of cells that always forms opposite the oncospheral hooks [8]. In classical studies of E. multilocularis metamorphosis, there is no indication of early anterior development and later regression, suggesting that anterior development is suppressed from the beginning [21]. Scattered studies of the development of metacestodes of the related Taenia spp. also indicate that they initially lack AP polarity, and develop as bladders from which one or more scolex primordia appear later [63, 72-76]. The lack of AP polarity during initial metacestode development, leading to a lack of correspondence between the polarity of the oncosphere and that of the metacestode, may be a characteristic of all taeniids.

Finally, the presence of muscle fibers in E. multilocularis metacestode vesicles has been enigmatic, since the fibers are disorganized and the vesicles lack motility $[21,24,77]$. The muscle system is probably homologous to that found in the bladder of other taeniids: in Taenia spp., the bladder tissue also has muscle fibers, is motile, and may assist in the evagination of the scolex $[17,24,78]$. Herein, we show that E. multilocularis muscle cells are a source of Wnt ligands, and suggest that this may account for the retention of this cell type in the otherwise immobile metacestode.

\section{Conclusions}

The unique biology of tapeworms has been a source of fascination and speculation, but their uniqueness has also confounded our ability to compare them to other animals and has hampered our understanding of their evolutionary origins. Identification of a conserved developmental stage in tapeworms opens the possibility to perform meaningful comparisons with free-living flatworms and other phyla for the first time. Conservation of the flatworm stem cell system and of underlying gene regulatory networks suggests that reciprocal illumination will come from studies of both planarians and parasitic flatworms [79].

\section{Methods}

Maintenance, culture and collection of tapeworms

E. multilocularis isolates were maintained by serial intraperitoneal passage in Meriones unguiculatus as previously described [80]. The isolates used were GH09 and J2012, obtained from accidental infections of Old World Monkeys in a breeding enclosure [81], and MS10/10, obtained from an infected dog. All isolates had been passaged for 5 years or less at the time of this study. Animal experiments were carried out in accordance with European and German regulations on the protection of animals (Tierschutzgesetz). Metacestode vesicles obtained from in vivo culture were co-cultured in vitro with rat Reuber hepatoma cells as previously described [80]. After at least 2 months of in vitro culture, metacestode vesicles began the development of brood capsules and protoscoleces, and were collected and fixed for WMISH and immunohistofluorescence with $4 \%$ paraformaldehyde prepared in phosphate buffered saline following the method of Koziol et al. [50]. Completely developed protoscoleces were isolated from parasite material maintained in vivo, and activated by successive treatments with pepsin at low $\mathrm{pH}$ and sodium taurocholate [82].

The Nottingham strain of $H$. microstoma [46] was maintained in vivo using outbred BALB/c mice and flour beetles (Tribolium confusum). Infection of beetles was produced by exposing them for 24 hours to macerated gravid tissues of $H$. microstoma. Then, beetles were allowed to feed on flour and metacestodes at different stages of metamorphosis were obtained by dissection on successive days. Larvae were fixed with paraformaldehyde prepared in phosphate buffered saline, as previously described [50].

\section{Molecular cloning}

Genes of interest were identified by BLAST searches against the genomes and derived gene models of $E$. $m u l$ tilocularis and H. microstoma [29] followed by phylogenetic analyses (Additional files 1, 2 and 3). Fragments of the coding domain sequences of $E$. multilocularis genes were amplified by RT-PCR using pools of cDNA from in vitro cultured metacestode vesicles, primary cells and protoscoleces, and cloned into pDrive (Qiagen, Hilden, Germany) or pJet1.2 (Thermo Scientific, Schwerte, Germany) following standard procedures, as previously described [50]. 
Hymenolepis transcripts were amplified using cDNA from larval or adult specimens and cloned into StrataClone vectors (Agilent Technologies, La Jolla, CA, USA). Colonies were picked and checked for insert directionality via PCR, then used as templates for PCR amplification using M13 primers, resulting in products with T3/T7 promoter sites that were purified and used subsequently as templates for reverse transcription. A list of all gene model codes and primers used in this work is provided in Additional file 6 .

\section{Whole-mount in situ hybridization (WMISH)}

Tyramide-FITC-based fluorescent and alkaline phosphatasebased conventional WMISH of $E$. multilocularis and $H$. microstoma larval stages were performed with digoxigeninlabeled antisense probes, as previously described [50]. E. multilocularis samples were analyzed by confocal microscopy using a Leica TCS SP5 (Leica Microsystems, Wetzlar, Germany). All gene expression patterns reported in this work from E. multilocularis metacestodes were obtained from at least two different WMISH experiments, starting from independent batches of in vitro cultured animals, with at least five metacestode vesicles analyzed in each experiment. Those of Hymenolepis are based on multiple independent assays, each containing approximately 10 in vivo-reared specimens of each larval stage (approximately 50 larvae per tube). Alkaline phosphatase-based WMISH specimens were imaged using differential interference contrast microscopy on a Leica DM5000B light microscope. Fluorescent specimens were imaged using a Nikon A1 confocal microscope and maximum projections created using ImageJ v. 2 [83]. Control experiments using labeled sense probes were always negative (data not shown).

\section{In vitro labeling with 5-ethynyl-2'-deoxyuridine (EdU)}

In vitro labeling with $50 \mu \mathrm{M}$ EdU was done for 5 hours and fluorescent detection with Alexa Fluor 555 azide was performed after WMISH following the method described in by Koziol et al. [50].

\section{Immunohistofluorescence}

The immunohistofluorescence procedure described by Koziol et al. [24] was carried out after WMISH detection. Anti-HMW-tropomyosin [53] was used as primary antibody at a 1:500 dilution. The secondary antibody was anti-rabbit conjugated to tetramethylrhodamine (Jackson ImmunoResearch, West Grove, PA, USA).

\section{Additional files}

Additional file 1: Phylogeny of Frizzled receptors. Frizzled receptors from H. sapiens, D. melanogaster, the trematode S. mansoni [84], the planarian S. mediterranea [84], and the tapeworms E. multilocularis and
H. microstoma [29] were aligned, and a phylogeny was estimated by Maximum Likelihood analysis (with a JTT model) using MEGA 5.2 [85]. The tree was rooted using the related Smoothened receptors. Bootstrap support values from 1,000 replicates are indicated next to the nodes. Nodes with lower than $50 \%$ support were collapsed. Genbank accession codes are given for $\mathrm{H}$. sapiens and D. melanogaster; GeneDB accession codes are given for $E$. multilocularis and H. microstoma; gene names from [84] are given for S. mansoni and S. mediterranea. The fz 4 genes of E. multilocularis and H. microstoma are indicated by arrows. (PDF $9 \mathrm{~kb}$ )

Additional file 2: Phylogeny of sine oculis-homeobox (SIX-HD) transcription factors. The homeodomain (HD) and SIX domains of SIX-HD proteins of H. sapiens, D. melanogaster, E. multilocularis, and H. microstoma were aligned together with published planarian SIX-HD proteins, and a phylogeny was estimated by Maximum Likelihood analysis (with a JTT model) using MEGA 5.2 [85]. Bootstrap support values from 1,000 replicates are indicated next to the nodes. Genbank accession codes are given for $\mathrm{H}$. sapiens, D. melanogaster, and for the planarians S. mediterranea and G. tigrina; GeneDB accession codes are given for E. multilocularis and H. microstoma. Different groups of SIX-HD proteins are outlined, and the six $3 / 6$ genes of E. multilocularis and H. microstoma are indicated by arrows. (PDF $109 \mathrm{~kb}$ )

Additional file 3: Phylogeny of Forkhead (FOX) transcription factors. The FKH domain of forkhead proteins of $H$. sapiens, D. melanogaster, E. multilocularis, H. microstoma, and published FoxQ2 homologs from other animals were aligned, and a phylogeny was estimated by Maximum Likelihood analysis (with a JTT model) using MEGA 5.2 [85]. Bootstrap support values from 1,000 replicates are indicated next to the nodes. Nodes with lower than $50 \%$ support were collapsed. GeneDB accession codes are given for E. multilocularis and H. microstoma and Genbank accession codes are given for all other sequences. The FoxQ2 group is outlined, and the foxQ2 genes of E. multilocularis and H. microstoma are indicated by arrows. (PDF $33 \mathrm{~kb}$ )

Additional file 4: Alkaline phosphatase-based development of whole-mount in situ hybridization in H. microstoma: SFRPs and

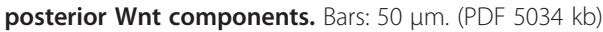

Additional file 5: Alkaline phosphatase-based development of whole-mount in situ hybridization in H. microstoma: Wnt2 and Wnt5. Bars: 50 um. (PDF 1653 kb)

Additional file 6: List of genes studied in this work. The GeneDB codes (www.genedb.org) of E. multilocularis and H. microstoma gene models are given, together with the primer sequences used for transcript amplification by RT-PCR. (XLSX $12 \mathrm{~kb})$

\section{Abbreviations}

AP: Antero-posterior; EdU: 5-ethynyl-2'-deoxyuridine; PCG: Position control gene; TPM: Tropomyosin; WMISH: Whole-mount in situ hybridization.

\section{Competing interests}

The authors declare that they have no competing interests.

\section{Authors' contributions}

UK conceived of the study, performed all experiments in E. multilocularis, and drafted the manuscript. FJ performed experiments in H. microstoma. PDO and $\mathrm{KB}$ participated in the design and supervision of the study and helped draft the manuscript. All authors read and approved the final manuscript.

\section{Acknowledgements}

This work was supported by grants from the Deutsche Forschungsgemeinschaft (DFG; BR 2045/4-1) and the Wellhöfer Foundation (all to KB). UK was supported by a grant of the German Excellence Initiative to the Graduate School of Life Sciences, University of Würzburg, and by a travel grant of the Comisión Sectorial de Investigación Científica (CSIC), Universidad de la República, Uruguay. FJ was supported by a doctoral training grant from the Natural History Museum and University College London Centre for Genomics, Ecology and Evolution (UK). The funding bodies had no role in the collection, analysis and interpretation of data, or in the writing of the manuscript and the decision to submit the manuscript for publication. The authors wish to thank Monika Bergmann and Dirk Radloff for their excellent technical assistance. 


\section{Author details}

${ }^{1}$ Sección Bioquímica, Facultad de Ciencias, Universidad de la República, Montevideo, Uruguay. ${ }^{2}$ University of Würzburg, Institute for Hygiene and Microbiology, Würzburg, Germany. ${ }^{3}$ Department of Life Sciences, The Natural History Museum, London, UK.

Received: 29 December 2015 Accepted: 25 January 2016 Published: 4 March 2016

\section{References}

1. Littlewood DTJ. The evolution of parasitism in flatworms. In: Maule AG Marks NJ, editors. Parasitic Flatworms Molecular Biology, Biochemistry, Immunology, Physiology. Oxford: CABI Publishing; 2006.

2. Laumer $C E$, Hejnol A, Giribet $G$. Nuclear genomic signals of the 'microturbellarian' roots of platyhelminth evolutionary innovation. Elife. 2015;4. doi:10.7554/eLife.05503.

3. Olson PD, Tkach W. Advances and trends in the molecular systematics of the parasitic Platyhelminthes. Adv Parasitol. 2005;60:165-243.

4. Brooks DR, Hoberg EP, Weekes PJ. Preliminary phylogenetic systematic analysis of the major lineages of the Eucestoda (Platyhelminthes: Cercomeria). Proc Biol Soc Wash. 1991;104:651-68.

5. Rybicka K. Embryogenesis in cestodes. Adv Parasitol. 1966;4:107-86.

6. Roberts LS, Davis RE. Platyhelminthes-Eucestoda. In: Adiyodi KG, Adiyodi RG, editors. Reproductive biology of invertebrates, vol IV, Part A: Fertilization, Development and Parental Care. Chichester: Wiley; 1989. p. 91-134.

7. Conn DB, Swiderski Z. A standardised terminology of the embryonic envelopes and associated developmental stages of tapeworms (Platyhelminthes: Cestoda). Folia Parasitol (Praha). 2008;55:42-52.

8. Freeman RS. Ontogeny of cestodes and its bearing on their phylogeny and systematics. Adv Parasitol. 1973;11:481-557.

9. Stunkard HW. The organization, ontogeny, and orientation of the Cestoda. Quart Rev Biol. 1962;37:23-34.

10. Olson PD. Hox genes and the parasitic flatworms: new opportunities, challenges and lessons from the free-living. Parasitol Int. 2008;57:8-17.

11. Gurley KA, Rink JC, Sanchez Alvarado A. Beta-catenin defines head versus tail identity during planarian regeneration and homeostasis. Science. 2008:319:323-7.

12. Almuedo-Castillo M, Sureda-Gomez M, Adell T. Wnt signaling in planarians: new answers to old questions. Int J Dev Biol. 2012;56:53-65.

13. Petersen $C P$, Reddien PW. Wnt signaling and the polarity of the primary body axis. Cell. 2009;139:1056-68.

14. Petersen CP, Reddien PW. Smed-betacatenin-1 is required for anteroposterior blastema polarity in planarian regeneration. Science. 2008;319:327-30.

15. Iglesias M, Gomez-Skarmeta JL, Salo E, Adell T. Silencing of Smedbetacatenin1 generates radial-like hypercephalized planarians. Development. 2008:135:1215-21

16. Cardona A, Hartenstein V, Romero R. Early embryogenesis of planaria: a cryptic larva feeding on maternal resources. Dev Genes Evol. 2006;216:667-81.

17. Slais J. Functional morphology of cestode larvae. Adv Parasitol. 1973;11:395-480.

18. Beveridge I. The use of life-cycle characters in studies of the evolution of cestodes. In: Littlewood DTJ, Bray RA, editors. The Interrelationships of the Platyhelminthes. London: Taylor \& Francis; 2001. p. 250-6.

19. Eckert J, Deplazes P. Biological, epidemiological, and clinical aspects of echinococcosis, a zoonosis of increasing concern. Clin Microbiol Rev. 2004:17:107-35.

20. Thompson RCA. Biology and systematics of Echinococcus. In: Thompson RCA, editor. The Biology of Echinococcus and Hydatid Disease. Crows Nest: George Allen \& Unwin; 1986. p. 5-43.

21. Sakamoto T, Sugimura M. Studies on echinococcosis XXIII. Electron microscopical observations on histogenesis of larval Echinococcus multilocularis. Jap J Vet Res. 1970;18:131-44.

22. Chervy L. The terminology of larval cestodes or metacestodes. Syst Parasitol. 2002;52:1-33

23. Thompson RC. The development of brood capsules and protoscolices in secondary hydatid cysts of Echinococcus granulosus. A histological study. Z Parasitenkd. 1976:51:31-6.

24. Koziol U, Krohne G, Brehm K. Anatomy and development of the larval nervous system in Echinococcus multilocularis. Front Zool. 2013:10:24.

25. Leducq R, Gabrion C. Developmental changes of Echinococcus multilocularis metacestodes revealed by tegumental ultrastructure and lectin-binding sites. Parasitology. 1992;104(Pt 1):129-41.
26. Goldschmidt R. Zur Entwicklungsgeschichte der Echinococcusköpfchen. Zool Jahrb. 1900:13:467-494.

27. Gurley KA, Elliott SA, Simakov O, Schmidt HA, Holstein TW, Sanchez Alvarado A. Expression of secreted Wnt pathway components reveals unexpected complexity of the planarian amputation response. Dev Biol. 2010;347:24-39.

28. Riddiford N, Olson PD. Wnt gene loss in flatworms. Dev Genes Evol. 2011:221:187-97.

29. Tsai IJ, Zarowiecki M, Holroyd N, Garciarrubio A, Sanchez-Flores A, Brooks KL, et al. The genomes of four tapeworm species reveal adaptations to parasitism. Nature. 2013;496:57-63.

30. Petersen CP, Reddien PW. Polarized notum activation at wounds inhibits Wht function to promote planarian head regeneration. Science. 2011;332:852-5.

31. Adell T, Salo E, Boutros M, Bartscherer K. Smed-Evi/Wntless is required for beta-catenin-dependent and -independent processes during planarian regeneration. Development. 2009;136:905-10.

32. Petersen CP, Reddien PW. A wound-induced Wnt expression program controls planarian regeneration polarity. Proc Natl Acad Sci U S A. 2009:106:17061-6.

33. Blassberg RA, Felix DA, Tejada-Romero B, Aboobaker AA. PBX/extradenticle is required to re-establish axial structures and polarity during planarian regeneration. Development. 2013;140:730-9.

34. Yazawa S, Umesono Y, Hayashi T, Tarui H, Agata K. Planarian Hedgehog/ Patched establishes anterior-posterior polarity by regulating Wnt signaling. Proc Natl Acad Sci U S A. 2009;106:22329-34.

35. MacDonald BT, He X. Frizzled and LRP5/6 receptors for Wnt/betacatenin signaling. Cold Spring Harb Perspect Biol. 2012;4(12). doi:10.1101/cshperspect.a007880.

36. van Amerongen R. Alternative Wnt pathways and receptors. Cold Spring Harb Perspect Biol. 2012:4(10). doi:10.1101/cshperspect.a007914.

37. Steinmetz PR, Urbach R, Posnien N, Eriksson J, Kostyuchenko RP, Brena C, et al. Six3 demarcates the anterior-most developing brain region in bilaterian animals. Evodevo. 2010;1:14

38. Pineda D, Salo E. Planarian Gtsix3, a member of the Six/so gene family, is expressed in brain branches but not in eye cells. Mech Dev. 2002:119 Suppl 1:S167-71.

39. Scimone ML, Kravarik KM, Lapan SW, Reddien PW. Neoblast specialization in regeneration of the planarian Schmidtea mediterranea. Stem Cell Reports. 2014;3:339-52.

40. Sinigaglia C, Busengdal $H$, Leclere $L$, Technau U, Rentzsch F. The bilaterian head patterning gene six $3 / 6$ controls aboral domain development in a cnidarian. PLoS Biol. 2013;11:e1001488.

41. Wei Z, Yaguchi J, Yaguchi S, Angerer RC, Angerer LM. The sea urchin animal pole domain is a Six3-dependent neurogenic patterning center. Development. 2009:136:1179-89.

42. Yu JK, Holland ND, Holland LZ. AmphiFoxQ2, a novel winged helix/forkhead gene, exclusively marks the anterior end of the amphioxus embryo. Dev Genes Evol. 2003;213:102-5.

43. Pearson JC, Lemons D, McGinnis W. Modulating Hox gene functions during animal body patterning. Nat Rev Genet. 2005;6:893-904.

44. Nogi T, Watanabe K. Position-specific and non-colinear expression of the planarian posterior (Abdominal-B-like) gene. Dev Growth Differ. 2001:43:177-84

45. Koziol U, Lalanne Al, Castillo E. Hox genes in the parasitic platyhelminthes Mesocestoides corti, Echinococcus multilocularis, and Schistosoma mansoni: evidence for a reduced Hox complement. Biochem Genet. 2009;47:100-16.

46. Cunningham LJ, Olson PD. Description of Hymenolepis microstoma (Nottingham strain): a classical tapeworm model for research in the genomic era. Parasit Vectors. 2010;3:123.

47. Voge M. Development of Hymenolepis microstoma (Cestoda: Cyclophyllidea) in the intermediate host Tribolium confusum. J Parasitol. 1964:50:77-80.

48. Witchley JN, Mayer M, Wagner DE, Owen JH, Reddien PW. Muscle cells provide instructions for planarian regeneration. Cell Rep. 2013;4:633-41.

49. Reuter M, Kreshchenko N. Flatworm asexual multiplication implicates stem cells and regeneration. Can J Zool. 2004:82:334-56.

50. Koziol U, Rauschendorfer T, Zanon Rodriguez L, Krohne G, Brehm K. The unique stem cell system of the immortal larva of the human parasite Echinococcus multilocularis. Evodevo. 2014;5:10.

51. Halton DW, Maule AG. Flatworm nerve-muscle: structural and functional analysis. Can J Zool. 2003;82:316-33. 
52. Koziol U, Costabile A, Dominguez MF, Iriarte A, Alvite G, Kun A, et al. Developmental expression of high molecular weight tropomyosin isoforms in Mesocestoides corti. Mol Biochem Parasitol. 2011;175:181-91.

53. Alvite $\mathrm{G}$, Esteves $\mathrm{A}$. Echinococcus granulosus tropomyosin isoforms: from gene structure to expression analysis. Gene. 2009;433:40-9.

54. Hartenstein $\mathrm{V}$, Jones $\mathrm{M}$. The embryonic development of the bodywall and nervous system of the cestode flatworm Hymenolepis diminuta. Cell Tissue Res. 2003;311:427-35.

55. Rohde K. The minor groups of parasitic platyhelminthes. Adv Parasitol. 1994:33:145-234.

56. Rohde K. Ultrastructural studies of Austramphilina elongata (Cestoda, Amphilinidea). Zoomorphology. 1986;106:91-102.

57. Olson PD, Littlewood DT, Bray RA, Mariaux J. Interrelationships and evolution of the tapeworms (Platyhelminthes: Cestoda). Mol Phylogenet Evol. 2001;19:443-67.

58. Martin-Duran JM, Egger B. Developmental diversity in free-living flatworms. Evodevo. 2012:3:7.

59. Martin-Duran JM, Amaya E, Romero R. Germ layer specification and axial patterning in the embryonic development of the freshwater planarian Schmidtea polychroa. Dev Biol. 2010;340:145-58.

60. Slack JM, Holland PW, Graham CF. The zootype and the phylotypic stage. Nature. 1993;361:490-2.

61. von Abzhanov A. Baer's law for the ages: lost and found principles of developmental evolution. Trends Genet. 2013;29:712-22.

62. Scimone ML, Lapan SW, Reddien PW. A forkhead transcription factor is wound-induced at the planarian midline and required for anterior pole regeneration. PLoS Genet. 2014;10:e1003999.

63. Bilqees FM, Freeman RS. Histogenesis of the rostellum of Taenia crassiceps (Zeder, 1800) (Cestoda), with special reference to hook development. Can J Zool. 1800;1969(47):251-61.

64. Swiderski Z, Miquel J, Mlocicki D, Georgiev BB, Eira C, Grytner-Ziecina B, et al. Post-embryonic development and ultrastructural characteristics of the polycephalic larva of Taenia parva Baer, 1926 (Cyclophyllidea, Taeniidae). Acta Parasitol. 1926;2007(52):31-50.

65. Hoberg EP, Jones A, Rausch RL, Eom KS, Gardner SL. A phylogenetic hypothesis for species of the genus Taenia (Eucestoda: Taeniidae). J Parasitol. 2000;86:89-98.

66. Knapp J, Nakao M, Yanagida T, Okamoto M, Saarma U, Lavikainen A, et al. Phylogenetic relationships within Echinococcus and Taenia tapeworms (Cestoda: Taeniidae): an inference from nuclear protein-coding genes. Mol Phylogenet Evol. 2011;61:628-38.

67. Lavikainen A, Haukisalmi V, Lehtinen MJ, Henttonen H, Oksanen A, Meri S. A phylogeny of members of the family Taeniidae based on the mitochondrial cox1 and nad1 gene data. Parasitology. 2008;135:1457-67.

68. Moore J, Brooks DR. Asexual reproduction in cestodes (Cyclophyllidea: Taeniidae): ecological and phylogenetic influences. Evolution. 1987;41:882-91.

69. Trouvé S, Morand S, Gabrion C. Asexual multiplication of larval parasitic worms: a predictor of adult life-history traits in Taeniidae? Parasitol Res. 2003:89:81-8.

70. Diaz A, Casaravilla C, Irigoin F, Lin G, Previato JO, Ferreira F. Understanding the laminated layer of larval Echinococcus I: structure. Trends Parasitol. 2011;27:204-13.

71. Rausch R. Studies on the helminth fauna of Alaska. XX. The histogenesis of the alveolar larva of Echinococcus species. J Infect Dis. 1954;94:178-86.

72. Mount PM. Histogenesis of the rostellar hooks of Taenia crassiceps (Zeder, 1800) (Cestoda). J Parasitol. 1970;56:947-61.

73. Heath DD, Smyth JD. In vitro cultivation of Echinococcus granulosus, Taenia hydatigena, T. ovis, T. pisiformis and T. serialis from oncosphere to cystic larva. Parasitology. 1970;61:329-43.

74. Heath DD, Elsdon-Dew R. The in vitro culture of Taenia saginata and Taenia taeniaeformis larvae from the oncosphere, with observations on the role of serum for in vitro culture of larval cestodes. Int J Parasitol. 1972;2:119-30.

75. Schramlova J, Blazek K. Morphology of Cysticercus bovis during its development. Folia Parasitol (Praha). 1983:30:335-9.

76. Bortoletti G, Ferretti G. Morphological studies on the early development of Taenia taeniaeformis larvae in susceptible mice. Int J Parasitol. 1985;15:365-75.

77. Lascano EF, Coltorti EA, Varela-Diaz VM. Fine structure of the germinal membrane of Echinococcus granulosus cysts. J Parasitol. 1975;61:853-60.

78. Slais J, Serbus C, Schramlova J. The microscopical anatomy of the bladder wall of Cysticercus bovis at the electron microscope level. Z Parasitenkd. 1971;36:304-20.
79. Collins 3rd JJ, Newmark PA. It's no fluke: the planarian as a model for understanding schistosomes. PLoS Pathog. 2013;9:e1003396.

80. Spiliotis M, Lechner S, Tappe D, Scheller C, Krohne G, Brehm K. Transient transfection of Echinococcus multilocularis primary cells and complete in vitro regeneration of metacestode vesicles. Int J Parasitol. 2008;38:1025-39.

81. Tappe D, Brehm K, Frosch M, Blankenburg A, Schrod A, Kaup FJ, et al. Echinococcus multilocularis infection of several old world monkey species in a breeding enclosure. Am J Trop Med Hyg. 2007;77:504-6.

82. Gelmedin V, Spiliotis M, Brehm K. Molecular characterisation of MEK1/2- and MKK3/6-like mitogen-activated protein kinase kinases (MAPKK) from the fox tapeworm Echinococcus multilocularis. Int J Parasitol. 2010:40:555-67.

83. Abramoff MD, Magalhaes PJ, Ram SJ. Image processing with ImageJ. Biophotonics Intern. 2004:11:36-42.

84. Zamanian M, Kimber MJ, McVeigh P, Carlson SA, Maule AG, Day TA. The repertoire of $\mathrm{G}$ protein-coupled receptors in the human parasite Schistosoma mansoni and the model organism Schmidtea mediterranea. BMC Genomics. 2011;12:596.

85. Tamura K, Peterson D, Peterson N, Stecher G, Nei M, Kumar S. MEGA5: molecular evolutionary genetics analysis using maximum likelihood, evolutionary distance, and maximum parsimony methods. Mol Biol Evol. 2011;28:2731-9.

\section{Submit your next manuscript to BioMed Central and we will help you at every step:}

- We accept pre-submission inquiries

- Our selector tool helps you to find the most relevant journal

- We provide round the clock customer support

- Convenient online submission

- Thorough peer review

- Inclusion in PubMed and all major indexing services

- Maximum visibility for your research

Submit your manuscript at www.biomedcentral.com/submit
) Biomed Central 